\title{
PUBLISH/SUBSCRIBE IoT SMART HOME
}

\author{
OCTAVIO JOSÉ SALCEDO PARRA ${ }^{1,2}$, SEBASTIAN R. MEDINA C. ${ }^{2}$ \& MIGUEL J. ESPITIA R. ${ }^{1}$ \\ ${ }^{I}$ Faculty of Engineering, Universidad Distrital Francisco José de Caldas, Bogotá, Colombia. \\ ${ }^{2}$ Faculty of Engineering, Universidad Nacional de Colombia, Bogotá D.C., Colombia.
}

ABSTRACT
Cloud Computing is increasingly intertwined with IoT, facilitating its implementation in both simple systems, as
well as in large distributed systems. Due to the advance in the development of APIs for IoT that currently provide
well-defined systems for the visualization, control and configuration of devices (temperature and humidity sensors,
light regulation and door management) via Cloud Computing, it will be shown how to implement a Smart model
Home using a Software Publish / Subscribe architectural design pattern from MOM (Message Oriented
Middleware) in which it is possible to distribute messages to different platforms, reducing the complexity of
implementing applications that extend to different operating systems and network protocols by unifying them.,
allowing a simple execution of Publish / Subscribe due to the level of abstraction and additional isolation provided
by the APIs. The implementation of a Smart Home totally based on IoT and Publish / Subscribe will be shown,
the different advantages of this approach will be described and the differences of this approach with Smart Homes
that use domotics as their main source of development will be studied.
KEYWORDS: API, Cloud Computing, IoT \& Smart Home

Received: Oct 04, 2020; Accepted: Oct 24, 2020; Published: Nov 21, 2020; Paper Id.: IJMPERDOCT202057

\section{INTRODUCTION}

It will show how to implement a system based on IoT and Cloud Computing to convert a common home into a smart home, which is interconnected through an SBC (Single Board Computer or Reduced Board Computer) to a service based on Cloud and IoT (Publish- Subscribe). The implementation process and examples of implementation of additional functionalities will be shown. In the development, two Cloud Computing services will be used: PubNub and Cayenne, which allow a simple interconnection of the SBC device with IoT functionalities. These services also provide high customization and different aids in the creation of user interfaces for communication and interaction with IoT devices either via web or via mobile. Python will be used as the implementation language, with the use of Adafruit libraries to control sensors and PubNub libraries to enable the use of Publish-Subscribe based on messages.

We have dedicated ourselves in the last century to simplifying all our daily tasks. Since the invention of the computer, all the processes we carry out day by day have been reduced to few simple instructions on a computer. On the last 3 decades, every family's dream was to have a home intelligent, which can have automated processes that make life easier, this became possible with home automation. With this technique focused on automating a home, could achieve a totally intelligent house, managed from some screen physically installed in the house. The problem of this technology is its high cost, requiring wiring special structured, devices and proprietary software, as well as its high difficulty of implementation in a home already built. 
The advantage of the Internet in its ease of interconnecting devices allows the development of state of the art techniques, there is no longer the need for structural cabling, proprietary devices and expensive software. With the new wave of IoT, where every device is interconnected and Cloud Computing that gives us immense computational power, we can implement a smart home at low cost, with little experience and fast. To get into context, the Publish / Subscribe design pattern will be explained:

It is a messaging pattern in software architecture, where the entities that send messages, called publishers, do not send messages to specific recipients. Instead it sends the characterized messages without needing to know if any subscriber needs to receive the message. In the same way, subscribers know what type of messages they are interested in receiving without knowing who the publisher is. This design pattern provides different advantages: ubiquity, network scalability, more dynamic network topology, and broadcast message support. This pattern is also a clear example of the MVC architecture.

\section{PREVIOUS STUDIES}

Different approaches were studied regarding the realization of Smart Homes based on IoT, it was observed that different implementations were concerned about the synchronization of the connectivity of the house, since different devices used different protocols to be interconnected, all this is solved by research and new programming models for IoT applications such as patRICIA [1], and systems defined by software for IoT. As Vittorio Miori and Dario Russo point out in their paper: "Domotic evolution towards the IoT" the problem of smart homes in the last decade lies in the difficulty of interconnection and the use of protocols, software and proprietary hardware: "Home automation is an extremely appealing application of the Internet of Things. It envisions a future home environment where embedded sensors and actuators (e.g., in consumer electronic products and systems) are selfconfiguring and can be controlled remotely through the Internet, enabling a variety of monitoring and control applications.

A solution proposed by Joseph Boman is to integrate middleware with IoT, so that standardized and user-friendly solutions can be developed; For this, in his research, he develops a data interpreter for IoT, which together with a Cloud service and an open source middleware manages to create one of the first ubiquitous middlewares for a simpler IoT development: "IoT must be supported by a middleware that allows IoT consumers and IoT application developers to interact in a user-friendly way, despite the differences in each user's perspective of IoT system. To that end, our software attempts to bridge the gap between IoT consumers and IoT application developers. Through the coupling of GSN (an existing open source IoT middleware), Firebase (a cloud storage service), and an IoT data interpreter developed by us, we have created a software system that takes the first step towards an ubiquitous middleware for IoT. " [2]. With advances in the development of APIs, the implementation or development of a proprietary middleware to be able to interact with IoT devices is not necessary, the Cloud services that play a vital role in the interconnection of IoT provide the necessary tools to be able to manage, configure and observe IoT devices, without the need for middleware or extra software to the one already provided by the IoT Cloud API, for this reason the implementation of the smart Home via Publish / Subscribe and PubNub is simple, familiar and user-friendly, as well as a clear implementation of the proposal of the authors of the paper: "Flexible IoT middleware for integration of things and applications" [2] with the difference of already being implemented together by the Cloud service, leading to an exponential decrease in the programming effort and difficulty of implementation .

Technology is increasingly moving away from physical computing power on site, due to its high cost, maintenance problems and low confidence due to its variable and unpredictable uptime. IoT is not the exception, the development of smart homes was possible but as Xiaojing Ye describes, Cloud Computing has reduced the difficulty of implementation and the 
maintenance and uptime problems of Smart Homes services, these being mission critical: "Although home automation technologies have been commercially available already, they are basically designed for signal-family smart homes with a high cost, and along with the constant growth of digital appliances in smart home, we merge smart home into smart-homeoriented Cloud to release the stress on the smart home system which mostly installs application software on their local computers. "[3] This approach will be used, where the stress of computing power, uptime and energy consumption is delegated to the IoT Cloud, facilitating processes, energy consumption and using the benefits of the internet.

All these aforementioned advances have been of great importance for the expansion of IoT, being applied mostly by cloud service companies for IoT such as Amazon Web Services, Microsoft Azure and SoftLayer. The innovation of PubNub, the IoT Cloud service based on Publish / Subscribe, relies on the ease of use of IoT devices; In addition to being these systems defined by software as in the other Cloud services, PubNub provides analysis, management and reading in real time, based on sending and receiving messages.

Being advantageous by the very nature of message-based programming: ubiquity, ease of programming, independent and granular handling of each function of the IoT device, all being unified in an API with free easy-to-implement libraries.

This is the main weakness observed in different implementations of IoT for a smart home: different communication protocols, proprietary software and hardware, difficulty in implementation, and difficulty in managing ubiquity. We will try to solve these problems through PubNub and Publish / Subscribe.

\section{METHODOLOGY}

Given the abstraction provided by the new definition of IoT by software, it will be possible to implement and control the home in a simple way, being the Cloud-based IoT service a facilitator, providing well-defined APIs that allow to put aside the difficulties of implementation to low level, as well as allowing access to dynamic resources and granular access to functionalities.

As a first step, the Python programming process will be explained to allow connection to the Cloud IoT service through Observer or Publish-Subscribe, as well as the configuration of hardware devices connected to GPIO and PWM, two enabling technologies of the SBC.

Cloud providers of IoT services generate software-based solutions by isolating all low-level component controls in their platform and infrastructure, unifying them in a well-defined access interface, similar to the Facade design pattern as can be seen in Figure 1. 




Figure 1: IoT Cloud platform architecture. Source: [4]

\subsection{Code Section}

It will describe the process of installing the necessary libraries in the SBC, examples of Python code to carry out PublishSubscribe and handling of GPIO ports. The simplicity in the coding of the SBC so that it can respond to the IoT Cloud is a fundamental part of the solution. The implementation has to be simple enough to be widely accepted in most homes. By leaving the hardware configuration process behind, the difficulty decreases. Another advantage of the implementation is thanks to the Observer design pattern, which allows asynchronous use of resources, further reducing the programming difficulty because the IoT Cloud, thanks to Observer, can receive asynchronous calls and execute different instructions at the same time without problem, all this without worrying about the difficulty of implementing parallel computing.

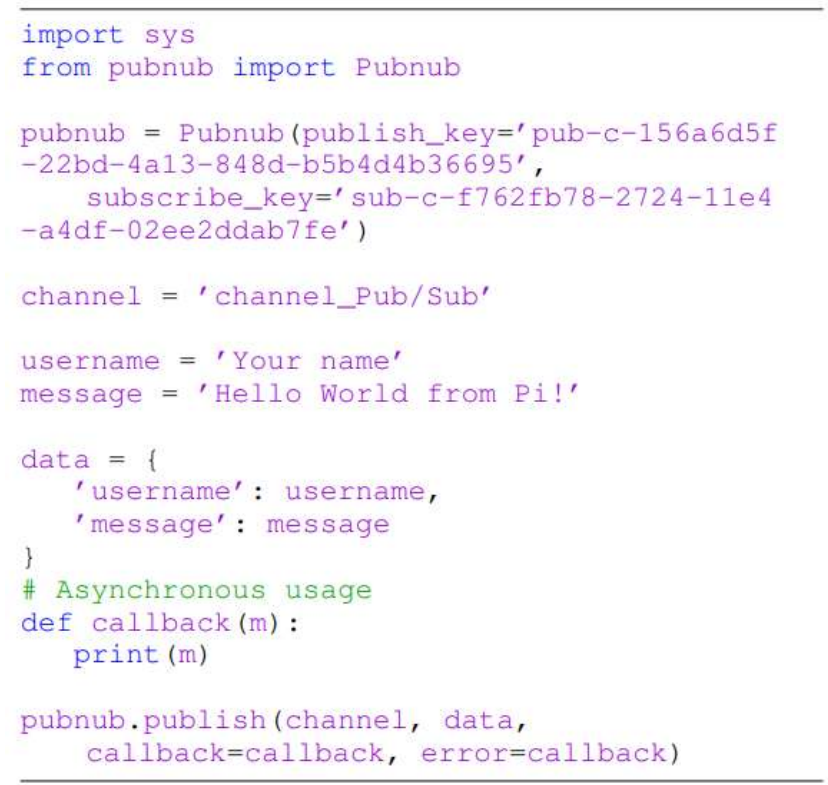

\section{Algorithm 1.}

With asynchronous Publish-Subscribe, and use of the PubNub library, the SBC will be connected with the IoT cloud unifying all IoT functions. 


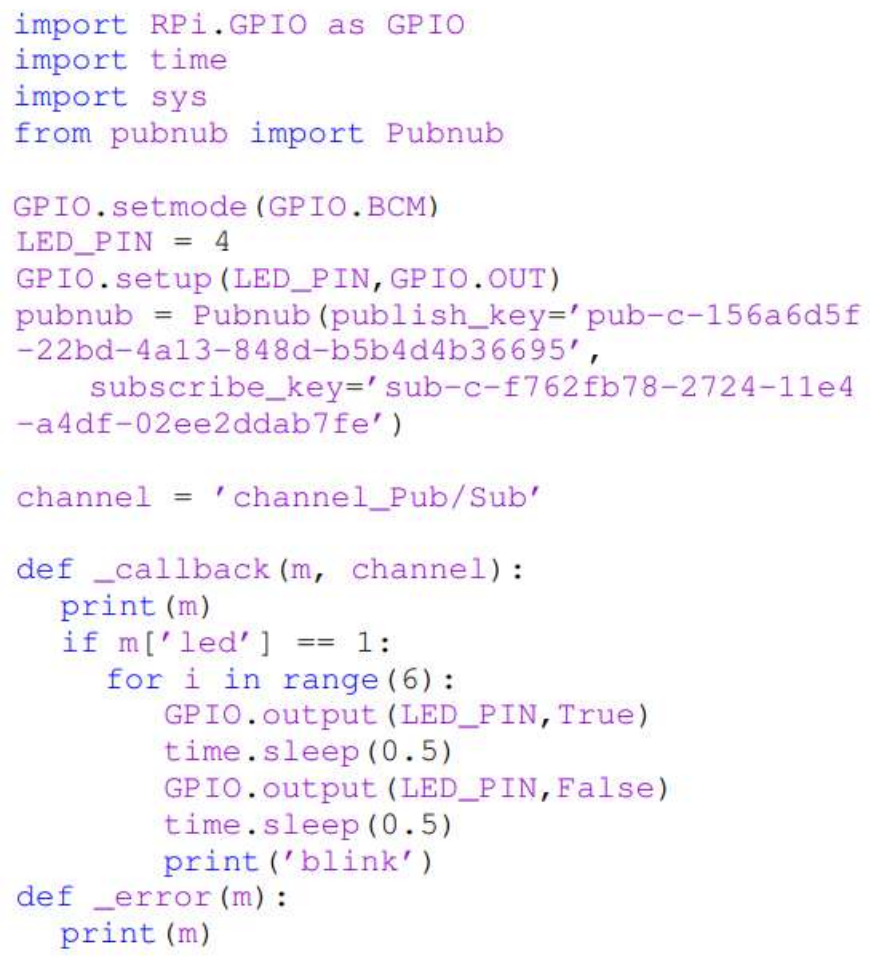

\section{Algorithm 2.}

Publish / Subscribe keys will be used to interconnect the SBC with the IoT Cloud, and the RP i.GP IO library will be used, in addition all the control of the smart home will be carried out from the Web, injecting JavaScript code to the page so that it is can communicate with the IoT Cloud and thus be able to manage the SBC remotely.

In the website to be developed, the Pubnub JS SDK will be injected and through this it will be connected through Publish / Subscribe to the IoT Cloud. 


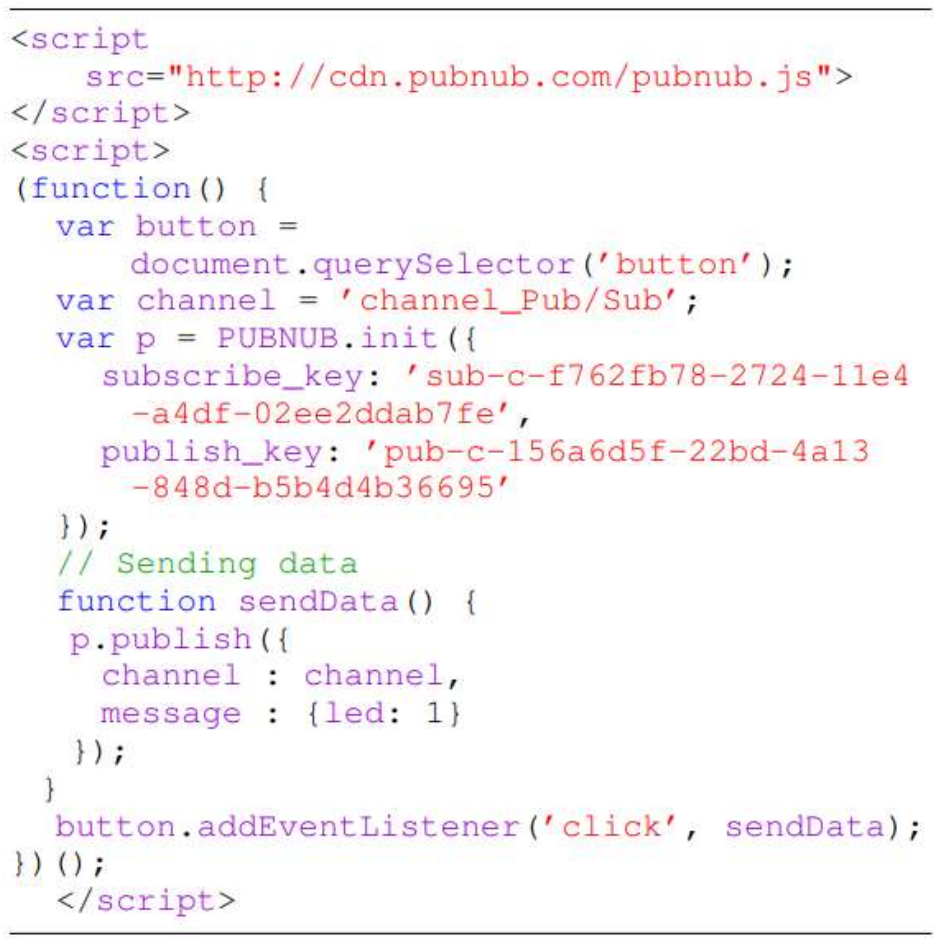

\section{Algorithm 3.}

In this way, the entire coding part will be implemented, it should be noted that the programming of events and their sending to the IoT Cloud is quite simple and does not require previous experience in advanced programming, facilitating its wide implementation in homes.

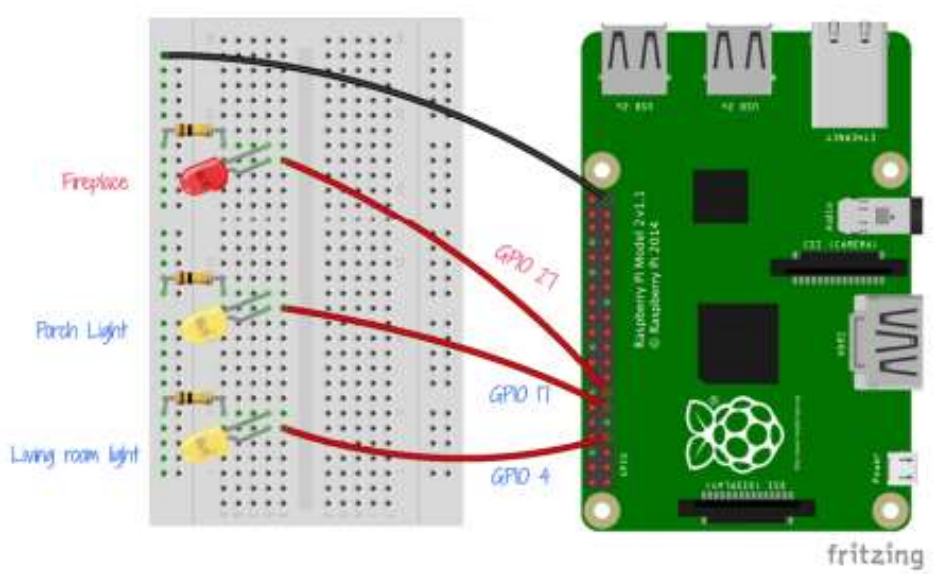

Figure 2: Basic circuit for connecting lights. Source: Authors

\section{IMPLEMENTATION}

The implementation of this project is carried out by means of a Raspberry Pi 3, breadboards, LEDs, DHT11 Sensor for Humidity and temperature and a SG90 Servo motor for the movement of doors. 


\subsection{Circuit}

A simple circuit is made that interconnects all the smart home devices with the SBC.

\subsection{Lighting}

It begins by generating the code for light management: where the GPIO and PWM library is used to be able to carry out an adequate management of LEDs. This management code will be subscribed using the Publish / Subscribe keys and a specific channel, in this channel the requests made from the web page that manages the smart home will be heard. Depending on the content of the message, it will perform different actions at the GPIO level on the LEDs, simulating an increase or decrease in brightness, as well as turning the lights on and off; These messages are sent using JavaScript and its defined PubNub API.

\subsection{Sensor for HVAC readings}

For the implementation of the DHT11 temperature and humidity sensor, a process similar to lighting will be carried out, with the difference of being a publisher, not a subscriber. This means that the code will publish the humidity and temperature readings to the web page, updating the values in real time. This portion of code will perform the control of the DHT11 sensor, process the temperature and humidity readings, the message format will be made, adding the two readings and will be published through PubNub. The web page will receive the data since it will subscribe to the same channel and will update the values in real time:

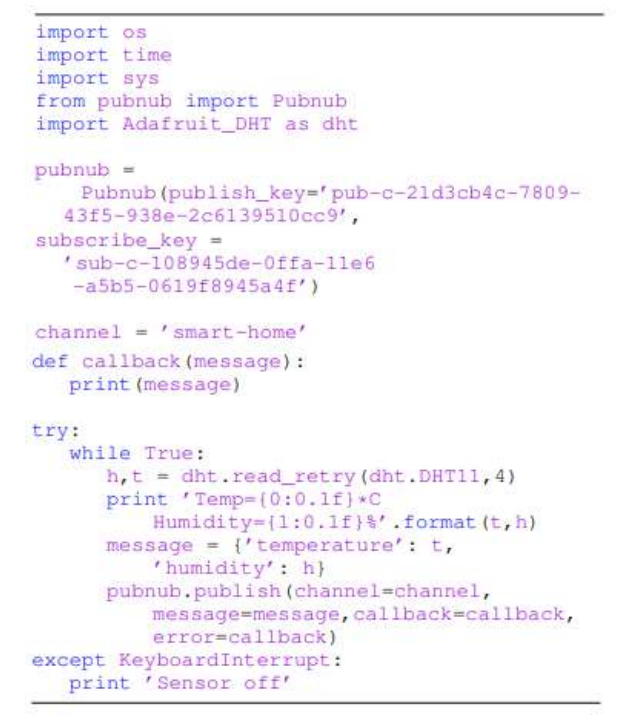

\section{Algorithm 4.}

\subsection{Servo Motor for automated door handling}

Servo implementation is quite simple: the motor subscription is performed in the same way as the previous functionalities, this being a subscriber that will receive one of two possible messages: True or False. Depending on the message that is sent from the web page the engine will rotate according to the message. It should be noted that this type of motor does not have continuous rotation, its movement depends on pulsations in a certain band of wave amplitude, in this case $50 \mathrm{~Hz}$. Due to its analogous nature, the servo motor tends to have jitter due to electronic noise, which is not a major problem but generates small erratic movements in the motor when it is still, either in the open or closed state. Below you can see the implementation of the servo motor using GPIO and PWM and subscribing it through PubNub: 


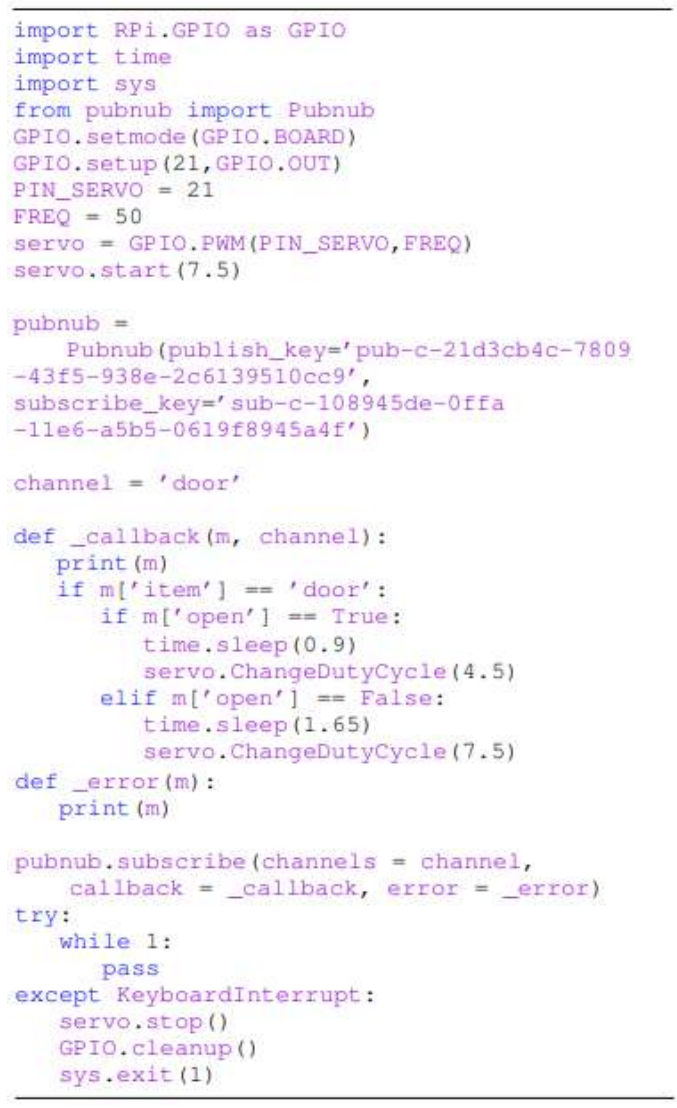

\section{Algorithm 5.}

\section{DISCUSSION OF RESULTS}

The functionalities of the IoT Smart Home are fast, efficient and low energy consumption, with respect to different explored investigations it can be said that this implementation is a simple way to make a low-cost and fast smart home. However, if you want a complete implementation in a home of considerable size, you must include more variables in the implementation, such as wiring, SBC cooling and more security in the front end of the web user interface, since any person can enter the website, for a large implementation the access system to the website must be ensured, as well as double-factor authentication to avoid system vulnerabilities as much as possible.

To improve the performance of the smart home and its accuracy, better quality sensors and actuators must be implemented, since low-cost sensors have a higher percentage of error. Sensors such as DHT22 for temperature and humidity control is more accurate, varying from $0 \%$ to $100 \%$ in range, while the one used in this implementation (DHT11) varies between $10 \%$ and $90 \%$, this taking into account that the DHT22 is worth the double.

According to different research published in the IEEE, it was possible to observe the rapid development of agile techniques for small and large-scale implementations of IoT, having advances such as those indicated by Vittorio Miori [5], Stefan Nastic [4] [6] and Joseph Boman [5] already implemented in the different IoT Cloud services, these being a fast integrator of new technologies that facilitate the expansion of IoT.

\section{CONCLUSIONS}

It was demonstrated how to achieve the automation of a home through IoT, with the special characteristic of being globally 
accessible, low costs, easy implementation, ubiquity in its processes and using the benefits of Cloud Computing. The final smart home model can be seen in Figure 3, where in operation it is possible to observe the management of lights, control of doors and real-time humidity and temperature readings shown on the website.

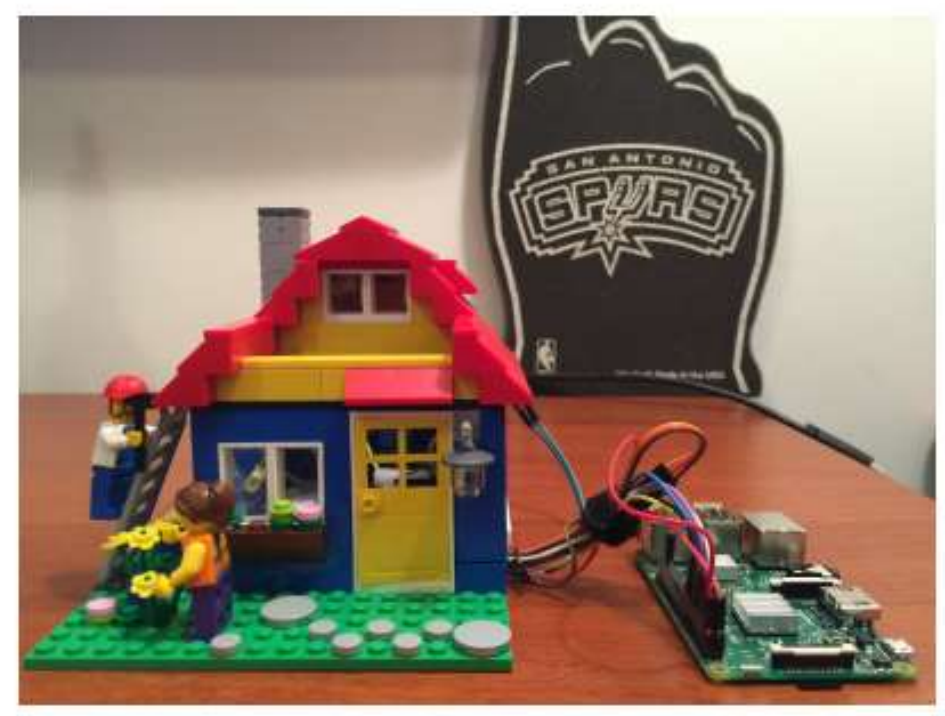

Figure 3: Smart Home model finished. Source: Authors

It can also be concluded that at the time of a more general and large implementation, where the interconnection of devices is not as trivial as in completely analog devices, the development is complicated and extensive, due to the difficulty of large-scale cabling and handling of concurrent messages in real time, this implies an increase in costs, since the free tiers in terms of Publish / Subscribe is around 1 million messages per month. When the number of messages is exceeded, which is very common to happen if If a home is interconnected with a greater number of devices, the costs of maintaining the smart home may increase.

Due to the remote management of home functionalities, this implementation can also be seen as a way to save on public utility costs, since it is possible to control and turn off lights or other devices with the purpose of reducing consumption without the need to be physically in home.

It could also be observed that this approach is applicable to a large extension of IoT projects due to the way of implementation, different approaches can be developed in a similar way, facilitating more complex projects based on IoT through the use of Publish / Subscribe. As extensions to the research, several scenarios can be proposed, such as implementing a solution that unifies automation through IoT not only of the Home but also the union of the Smart Home with the car, being able to control both entities through a common interface. Also measurement of services, consumption control, automatic grocery orders through the refrigerator, automatic payments of household expenses through the same interface and other functionalities that can make life easier.

\section{REFERENCES}

1. Stefan Nastic, Sanjin Sehic, Michael Vogler, Hong-Linh Truong, and Schahram Dustdar Distributed Systems Group, Vienna University of Technology, Austria. PatRICIA a Novel Programming Model for IoT Applications on Cloud Platforms.

2. Flexible IoT middleware for integration of things and applications. Joseph Boman; Jonathan Taylor; Anne H. Ngu. 
3. A framework for Cloud-based Smart Home Xiaojing Ye; Junwei Huang. Computer Science and Network Technology (ICCSNT), 2011 International Conference Communications, pp. 38

4. Stefan Nastic, Sanjin Sehic, Duc-Hung Le, Hong-Linh Truong, and Schahram Dustdar Distributed Systems Group, Vienna University of Technology, Austria. Provisioning Software-defined IoT Cloud Systems.

5. Domotic evolution towards the IoT, Vittorio Miori and Dario Russo Italian National Research Council.

6. PatRICIA - A Novel Programming Model for IoT Applications on Cloud Platforms Stefan Nastic; Sanjin Sehic; Michael Vgler; HongLinh Truong; Schahram Dustdar. 2013 IEEE 6th International Conference on Service-Oriented Computing and Applications. 